\title{
Challenges of Digital Transformation in Healthcare
}

\author{
Serik Smagulov, \\ Viktoriya Smagulova MD
}

\begin{abstract}
This article addresses the challenges of introducing digitalization technologies in healthcare. Connected devices mean infinite patient data collection. One of the main issues is the issue of security of personal data. This is a huge problem for all technology industries but more so for health care, given the personal nature of the data. Most healthcare organizations are so cautious about the consequences that they do not decide on a digital immersion. The article details the problems of cybersecurity and cyber attacks. Personal data security issues are particularly tangible in the field of the Internet of Things (IoT). There is another reason that puts most digital conversion plans to a standstill; this is a cost factor. Data aggregation and interoperability is another critical issue for healthcare institutions. Integration and interfaces, in this case, are unnecessarily complicated and risky. The author notes the importance of digital transformation, which should not only revolutionize patient care but also allow healthcare organizations to reduce the amount of time spent on documenting and data entry. And the data itself should be reliably protected.
\end{abstract}

Keywords: digitalization, cyber attacks, cybersecurity, Internet of things, database.

Connected devices mean infinite patient data collection. Electronic records help medical personnel reduce the time they need to search for information about each patient. Data collected from the patient's wearable device can also be safely stored in his electronic medical record, which makes it easy to diagnose when all the information is in one place. In this case, the main question still arises - the issue of personal data security.

Data security is also essential because of the increasing threat of cyber attacks. This is a massive problem for all technology industries, but more so for health care, given the personal nature of the data $[1 ; 2 ; 3]$. Most healthcare organizations are so cautious about the consequences that they do not decide on a digital immersion.

Embedding big data in healthcare significantly increases data privacy and patient safety. The main reason is that their information is stored in data centers with different levels of security. Moreover, data comes from different places, which carries additional risks.

A cloud solution that uses big data helps strengthen security to meet HIPAA requirements, which is unique to the healthcare industry. Also, it is a cost-effective solution. 
The rapid digitization of health care delivery, from electronic health records and telehealth to mobile health (mHealth) and network-enabled medical devices, introduces risks related to cybersecurity vulnerabilities [4].

Experts agree that for enterprises there are many ways to ensure the safety of patient data and research data in the digital environment. Cybersecurity should be a significant concern.

Organizations need to invest in staff with the right skill set, train employees in security measures, update known software vulnerabilities and ensure the safe construction of systems and processes. In an ever-changing digital world, you need to improve security measures in order to control cyber threats constantly.

Cyber attacks may also affect the safety of patients, for example, by compromising the integrity of data or impairing medical device functionality.

The WannaCry and NotPetya ransomware attacks and vulnerabilities in Medtronic Implantable Cardiac Device Programmers are recent examples that have resulted in impaired health care delivery capabilities [5].

Personal data security issues are particularly tangible in IoT.

The Internet of Things (IoT) has opened up a whole world of opportunities in medicine: when connected to the Internet, conventional medical devices can collect valuable additional data, provide further insights into symptoms and trends, provide remote treatment and generally give patients more control over their lives and treatments.

Wearable devices have become products that patients now carry, transferring data to doctors and, thus, allowing doctors to track vital signs in real time, including heart rate, glucose level, and even fall detection.

The introduction of the Internet of Things technology has created many problems regarding the privacy and security of personal data. Although many modern devices use secure methods to transfer information to the cloud, they may still be vulnerable to hackers.

In addition to theft and unauthorized use of personal data, IoT devices can be used to harm. To counter these risks, the US Food and Drug Administration (FDA) has published 
many guides to ensure the integrated safety of connected medical devices, and regulators are likely to continue to regulate the connected devices used by patients.

There is a complex structure of controllers and data processors with many different policies, privacy notices, and consents. The sensitivity of the data and the fact that most of their data is still stored on paper facilitates auditing, control, and access.

Often you can face to ransomware attacks. Finding employees with the right skill set, educating employees about security measures, updating known software vulnerabilities, and ensuring that systems and processes have a secure design is crucial to implementing more stringent security measures to eliminate the risks of cyber threats $[6 ; 7]$.

There is another reason that puts most digital conversion plans to a standstill; this is a cost factor. Medical organizations tend to focus on return on investment (ROI), not thoroughly evaluating the value proposition.

Some businesses find that adopting a digital strategy offers a larger scale, revenue, and profit. Instead of considering the costs incurred, it is better to take into account the money saved as soon as the inefficient processes are corrected by digital conversion. For example, telemedicine can reduce the need for a personal consultation, which allows physicians to serve more patients and, consequently, save money.

The same is true for the treatment of chronic diseases. Telemedicine can really save money $[8 ; 9 ; 10]$.

Data aggregation and interoperability is another challenge for healthcare institutions. The amount of data that health organizations generate daily is enormous, but very few can use it wisely.

When data is stored in an organization's repositories, it is difficult to access, analyze and work using available data. The problem can not be solved just adding a new technology which many enterprises have mistaken for digital transformation.

To make a holistic, patient-centered perspective a reality, health organizations should focus on integrating data from all systems and facilitate cross-functional 
collaboration. There should be a comprehensive solution to the problem, not imposing responsibility only in the IT department.

During participation in digitalization, many health institutions are confronted with a lack of an overall picture. Many hospitals claim to be "digital" thanks to the implementation of an integrated hospital information system (HIS) and electronic medical records (EMR). But this is not enough to be digital.

From registration and consultation to inpatient care and follow-up after discharge, the client's entire journey must be considered when planning a hospital's digital conversion. Unfortunately, very few institutions have a clear overall picture before they embark on the path of digitalization.

Companies must develop a clear vision of how they will meet the digital needs of their customers, set goals against this vision, and then begin with implementation. The process should be carried out, starting with investing in upgrading outdated systems and ending with offering real-time solutions and solving security problems.

In any case, when making the necessary changes, it is required to initially change the behavior of the person himself and the action of the management.

Changing a piece of equipment or even software is relatively easy compared to convincing people to change the way they work and spend time learning how to use new systems. This process should be carefully monitored to ensure that change for all is as easy to adapt as possible, and people interested in making changes.

People will use the new system only if they see the gap that it fills or the efficiency that it creates. Early-stage end-user participation is crucial to ensure that the technology meets their needs and that the user interface is convenient for them.

Sometimes in the process of implementing a digitalization program, there is a problem with interoperability for digital health systems. Integration and interfaces, in this case, are unnecessarily complicated and risky. 
Staying up to date with the latest technology and changing rules is crucial to improving processes and meeting the needs of patients in a changing digital world. Here both trends remain that need to be addressed in order to preserve health care in the 21st century.

The health care industry must take into account the pace of digital technology diffusion in various sectors. Health care companies must be significantly transformed to remain relevant.

The digital transformation should not only revolutionize patient care but also allow healthcare organizations to reduce the amount of time spent on documenting and data entry. And the data itself must be reliably protected.

\section{References}

1. Lynne Coventry, DawnBranley. Cybersecurity in healthcare: A narrative review of trends, threats and ways forward // Maturitas. Volume 113, July 2018, Pages 48-52.

2. Kruse CS, Frederick B, Jacobson T, Monticone DK. Cybersecurity in healthcare: A systematic review of modern threats and trends // Technological Health Care. 2017;25(1):1-10. doi: 10.3233/THC-161263.

3. Gordon W, Fairhall A, Landman A. Threats to Information Security - Public Health Implications. N Engl J Med 2017 Aug 24;377(8):707-709.

4. Jalali MS, Kaiser JP. Cybersecurity in Hospitals: A Systematic, Organizational Perspective. J Med Internet Res 2018 May 28;20(5):e10059

5. Furnell S, Emm D. The ABC of ransomware protection. Computer Fraud \& Security 2017 Oct;2017(10):5-11.

6. Kramer D, Fu K. Cybersecurity Concerns and Medical Devices: Lessons From a Pacemaker Advisory. JAMA 2017 Dec 05;318(21):2077-2078.

7. Jalali MS, Russell B, Razak S, Gordon WJ. EARS to cyber incidents in health care. J Am Med Inform Assoc 2019 Jan 01;26(1):81-90.

8. Michael Coakley, Giancarlo Crocetti. Transforming Telemedicine Through Big Data Analytics // semanticscholar.org. 
9. Vinayak K. Bairagi. Big Data Analytics in Telemedicine: A Role of Medical Image Compression // Big Data Management pp 123-160

10. Antonino Galletta, Lorenzo Carnevale, Alessia Bramant, Maria Fazio. An Innovative Methodology for Big Data Visualization for Telemedicine // IEEE Transactions on Industrial Informatics. Volume 5. Issue 1. 